\title{
Salienz und System
}

\section{Manfred Michael Glauninger (Wien)}

\begin{abstract}
The object of investigation in this paper is the linguistic conception of salience by focusing its still predominantly essentialistic, only in modest approaches constructivistic, modelling. This indicates one of the most basic methodological desiderata of linguistics: the discussion and revision of its systemic foundation. Therefore, the aim of this article is to initiate a decidedly constructivist discussion of linguistic salience, and its adaption and transformation, respectively. This could result in an exponentiated connectivity of (post-)modern constructivistic sociolinguistics to advanced self-referential autopoietic positions of systems theory.
\end{abstract}

\section{Gegenstand, Problem, Ziel}

Den Gegenstand dieses Beitrags bildet das linguistische Salienzkonzept unter Fokussierung seiner nach wie vor überwiegend essentialistischen, nur in bescheidenen Ansätzen konstruktivistische Aspekte aufweisenden Modellierung. Als essentialistisch ist - aus radikal bzw. operativ konstruktivistischer Sicht (cf. Baraldi/Corsi/Esposito 1997: 102) - jedwede Position zu bezeichnen, die Erkenntnis auf die Korrespondenz zwischen einem epistemologischen Subjekt und einem vermeintlich vorab existierenden, "im Sinne einer progressiven Enthüllung" (ibd.: 100) zu untersuchenden Objekt zurückführt. So gesehen weisen sämtliche etablierten linguistischen Paradigmen, d. h. insbesondere die gesamte Palette strukturalistischer und generativ fundierter Ansätze, sowie der darin eingebettete Salienzbegriff essentialistische Züge auf. ${ }^{1}$ Dieser - innerlinguistisch bezeichnenderweise meist unreflektierte - wissenschaftstheoretische Status stellt nicht nur per se ein Manko dar, er indiziert gleichzeitig eines der wohl grundlegendsten method(olog)ischen Desiderate der Sprachwissenschaft: die Diskussion bzw. Revision ihrer systemtheoretischen Basis. Letzteres gilt als übergeordnete Problemstellung der vorliegenden Arbeit, deren unmittelbares Ziel es jedoch ist, eine dezidiert und explizit konstruktivistische Diskussion des linguistischen Salienzbegriffs anzustoßen (cf. Auer 2007: 10). Daraus könnte eine einschlägige Adaption und Transformation des Salienzkonzepts resultieren sowie darüber hinaus eine punktuelle Steigerung der Anschlussfähigkeit der (post-)modernen, konstruktivistischen Soziolinguistik gegenüber innovativen, prozessual-selbstreferenziellen - und ebenfalls konstruktivistischen systemtheoretischen Positionen. ${ }^{2}$

\footnotetext{
1 Daran kann auch der - im Fall des Strukturalismus zum Teil gegebene - Input vonseiten (post-)moderner soziologischer Theorien bzw. ganz allgemein die Vorstellung von Sprache als fait social nichts ändern, wie etwa Schmaus (cf. 1994: 59) unter Verweis auf den Durkheim'schen Essentialismus vor Augen führt.

2 Dieses sowohl allgemein method(olog)isch und erkenntnistheoretisch als auch im engeren Sinn soziologisch bzw. (sozio-)linguistisch motivierte Vorhaben steht im Zentrum der Bildung einer "funktional dimensionierten" Sprachvariationstheorie (cf. Glauninger 2012), auf die im Folgenden mehrmals Bezug genommen wird.
}

Linguistik online 66, 4/14 - http://dx.doi.org/10.13092/lo.66.1570

licensed under CC 3.0 


\section{Das strukturalistische System als Fundament des linguistischen Essentialismus}

Es ist hier selbstredend nicht der Ort, um den Systembegriff der bekanntermaßen keinesfalls als einheitliches Theoriegebäude aufzufassenden, in einer Zeitspanne von mehreren Jahrzehnten Wissenschaftsgeschichte hervorgetretenen und auch in der Gegenwart beweglichen strukturalistischen (Haupt-)Strömungen innerhalb der Linguistik in extenso zu diskutieren, geschweige denn detailliert zu charakterisieren. Stattdessen soll ein kritischer Blick aus rezent systemtheoretischer bzw. konstruktivistisch-soziolinguistischer Perspektive auf folgende zwei - im Sinn des einleitend Festgehaltenen besonders aufschlussreiche Problembereiche geworfen werden: a) das Saussure'sche Langue-Modell vor dem Hintergrund seines naturwissenschaftlichen Vorbilds; ${ }^{3}$ b) die (im bzw. auf Basis des Cours postulierten) strukturalistischen Kriterien für Systematizität.

\subsection{Die langue als Adaption des prä-quantentheoretischen Strukturmodells der Materie}

Ein innerhalb der Sprachwissenschaft traditionell tief verankertes Verfahren besteht darin, bei grundlegenden Modellbildungen maßgebliche bzw. unmittelbare Anleihen zu nehmen von im jeweiligen historischen Kontext besonders erfolgreichen (und dabei idealiter in der breiten Öffentlichkeit populären) naturwissenschaftlichen Disziplinen. Auf diesen wissenschaftsgeschichtlich gut belegbaren (wenngleich $\mathrm{zu}$ wenig erforschten) sowie (wissenschafts-)soziologisch hinreichend erklärbaren Mechanismus der Orientierung an Leittheorien der zur gegebenen Zeit prestigeträchtigsten naturwissenschaftlichen Disziplinen gehen neben dem Saussure'schen ("Genfer") Strukturalismus (Näheres im Anschluss) u. a. wesentliche Züge der junggrammatischen Programmatik (in Form eines Pseudodarwinismus, cf. etwa Besch et al. 1998: 484; McMahon 1999: 19-21 und Raimy/Cairns 2009: 299) zurück, aber auch die anfänglich stark kybernetisch / informationstechnisch determinierte, später zunehmend und gegenwärtig fast durchgehend pseudo(gen)biologische Charakteristik des Generativismus. ${ }^{4}$ Im Fall der Valenzgrammatik wurde konsequenterweise zusammen mit dem grundlegenden Theorieansatz gleich auch dessen Name aus der Chemie übernommen.

Wie nun das im Cours tradierte Bild des Saussure'schen Langue-Konzepts eindrucksvoll veranschaulicht, muss der Einfluss, den das Strukturmodell der Materie der präquantentheoretischen Physik bzw. Chemie auf Saussure ausgeübt hat, entscheidend für dessen linguistisches Denken gewesen sein. So setzt er atomare Einheiten an (Phonem, Morphem, Graphem, ...), die - gewissermaßen molekular verbindungsfähig - das "System" Sprache "strukturiert-relational", in unterschiedlichen Ebenen geschichtet, aufbauen. Saussure, der am Beginn seiner akademischen Laufbahn an der Universität Genf zwei Semester lang Chemie und Physik inskribierte (cf. Bally/Sechehaye 2001: 294), hat die epochalen Umwälzungen innerhalb der Physik, die in den Jahren seiner - postum in den Cours eingehenden - Vorlesungen zur allgemeinen Sprachwissenschaft bereits im Gang waren, mit Sicherheit nicht mehr hinreichend rezipiert, ${ }^{5}$ sodass die langue an kein konstruktivistisches naturwissenschaftliches Modell

\footnotetext{
${ }^{3}$ Man könnte durchaus auch von einem naturwissenschaftlichen Original und seiner, gelinde gesagt, unbedarften linguistischen Kopie sprechen.

4 So konstatiert etwa Berg (1998: 5): "Chomsky's approach [...] may be characterized as a pseudobiological one". Deppermann (2007: 19) spricht von einer "letzten Endes biologistischen universalgrammatischen Auffassung der Generativisten". Schließlich reflektiere man in diesem Zusammenhang die folgende, unter der Überschrift "The study of language in a biological setting" firmierende generative Selbstpositionierung: "In this perspective, language is a natural object, a component of the human mind, physically represented in the brain and part of the biological endowment of the species" (Chomsky 2002: 1).

${ }^{5}$ Bzw. deren enorme Bedeutung und Tragweite wohl nicht abschätzen können, wofür ihm kein Vorwurf zu machen ist.
} 
angelehnt ist, ${ }^{6}$ sondern eine Interpretation der (mengentheoretisch-essentialistischen) Vorstellung von der Materie gemäß der "klassischen" Physik der Moderne darstellt. Es sei darüber hinaus nachdrücklich darauf hingewiesen, dass der Kernbereich der Saussure'schen Theorienbildung, die Modellierung der semiotischen Basis, aus heutiger Sicht im Hinblick auf seine explikative Reichweite bzw. Beschreibungsadäquatheit und Operabilität erheblich hinter dem Niveau der universalen - und deutlich konsequenter konstruktivistischen - Zeichentheorie seines Zeitgenossen Charles S. Peirce zurückblieb, dessen bedeutsamste Arbeiten Saussure aber aus überlieferungsgeschichtlichen Gründen nicht kannte. Gerade die semiotische Sphäre des linguistischen Strukturalismus erweist sich als besonders anfällig für essentialistische (Miss-)Deutungen. ${ }^{7}$ Dies liegt vor allem an der durchgehend adynamischen Justierung, die dem Saussure'schen Ideal des Panchronie-Verbots geschuldet ist (cf. Schmidt/Herrgen 2011: 21) und dazu verleitet, das (binär konfigurierte) sprachliche Zeichen - wenngleich als arbiträr und konventionell definiert - per se, gewissermaßen (in sich) ruhend, als (mental/kognitiv) existierend zu betrachten.

\subsection{Strukturalismus und Systematizität}

Aus systemtheoretischer Sicht (cf. Luhmann 1987: 15-29) repräsentiert das Systemkonzept des Strukturalismus, welches in seinen entscheidenden Zügen gegenwärtig nach wie vor nahezu allen tonangebenden, insbesondere dezidiert empirisch operierenden linguistischen Strömungen ${ }^{8}$ zugrunde liegt, ein Entwicklungsstadium, das durch zwei vor geraumer Zeit innerhalb der allgemeinen Systemtheorie vollzogene Paradigmenwechsel nachhaltig transformiert bzw. überwunden worden ist. Das strukturalistische System ist also, was seinen Status im Licht der Evolution der Systemtheorie anbelangt, ein überholtes Modell, welches letztlich der seit der Antike etablierten Vorstellung einer Ganzheit, die aus Teilen besteht, entspricht. ${ }^{9}$ In einem ersten Evolutionsschub wurde nun im Design der Systemtheorie "die traditionelle Differenz von Ganzem und Teil durch die Differenz von System und Umwelt ersetzt" (ibd.: 22). Entscheidend aber - und "von überbietender Radikalität" (ibd.) - fällt der daran anschließend vollzogene, zweite Paradigmenwechsel ins Gewicht, der zur Etablierung einer Theorie selbstreferenzieller, autopoietischer Systeme (cf. Baraldi/Corsi/Esposito 1997: 29-33, 163-167) geführt hat. Das strukturalistisch-essentialistische Schlüsselphänomen bzw. -kriterium für Systematizität, die Relation(alität) (zwischen sämtlichen Elementen innerhalb

\footnotetext{
6 Der Konstruktivismus kann in den "exakten" oder "strengen" Wissenschaften seit der Etablierung der Quantentheorie als state of the art bezeichnet werden: "Die Naturwissenschaft beschreibt und erklärt die Natur nicht einfach so, wie sie »an sich « ist. Sie ist vielmehr Teil eines Wechselspiels zwischen der Natur und uns selbst [...], dadurch wird eine scharfe Trennung zwischen der Welt und dem Ich unmöglich" (Heisenberg 1990: 60). S. auch Anmerkung 7.

7 Selbstverständlich weist die Saussure'sche Semiotik, ebenso wie sein Systemkonzept, Züge eines - aus gegenwärtiger Perspektive allerdings rudimentären bzw. prätheoretischen - Konstruktivismus auf, die sich in den Postulaten der Arbitrarität des Zeichens sowie der differenztheoretischen/differenzialistischen Relationalität bündeln. Schwer wiegt aus konstruktivistischer Sicht freilich das Manko, dass die Annahme einer Korrespondenz dieser und anderer "subjektiven" Phänomene mit einer externen, "objektiven" Wirklichkeit (s. oben, Abschnitt 1) beibehalten, zumindest nicht explizit in Frage gestellt wird, was sich letztlich in der spezifischen Konstellation von langue und parole am eindrucksvollsten widerspiegelt. Letzteres ist darüber hinaus als eine Art Platonismus zu klassifizieren, d. h. als eine Strategie der idealistischen Homogenisierung der Heterogenität des linguistischen Untersuchungsobjektes (natürliche) Sprache, der nahezu 1:1 die Korrespondenz zwischen Kompetenz und Performanz innerhalb generativer Theorien entspricht.

${ }^{8}$ Dazu zählen in der Regel keine generativen Paradigmen, deren überwiegend modular-komponentielle (und dabei nicht weniger essentialistische) Systemmodellierung allerdings sehr oft strukturalistische Einflüsse aufweist, respektive mit strukturalistischen Elementen zu hybriden Ansätzen fusioniert wird. Zur Berücksichtigung einer demgegenüber prozessual-autopoietischen Systemkonzeption im Rahmen der Bildung einer konstruktivistisch soziolinguistischen, funktional dimensionierten Sprachvariationstheorie s. Anmerkung 2 und weiter unten.

${ }^{9}$ Diese Konzeption wurde ab dem späten 19. Jahrhundert mengentheoretisch, d. h. als Menge-Element-Beziehung, interpretiert bzw. reformuliert.
} 
eines Systems) (cf. Payne/Barbera 2010: 668) ${ }^{10}$ wird dabei ersetzt durch konstruktivistischprozessuale Systematizitätsbedingungen, insbesondere jene der autopoietischen Operation(alität). Diese wird vom System ge(währ)leistet und beobachtet, und zwar systemintern. Zugleich sind diese prozessualen, selbstreferenziellen Operationen als solche das System. Auch das Kriterium der Funktionalität im Hinblick auf Komplexitätsreduktion (in unterschiedlichster Ausprägung) spielt eine entscheidende Rolle. Aus dem Festhalten der Linguistik an ihrer überholten systemtheoretischen Basis resultieren eine Reihe von handgreiflichen Problemen. An dieser Stelle seien - in gebotener Komprimierung - zwei davon herausgegriffen. Erstens impliziert das Saussure'sche Postulat der Relationalität als Konstituens von Systematizität (vereinfacht ausgedrückt: In einem System sind alle Elemente in bestimmter Weise miteinander verbunden/verknüpft, was letztlich auf die essentialistische Vorstellung einer Struktur hinausläuft) eine nicht operationalisierbare Komplexität ${ }^{11}$ und steht darüber hinaus im Widerspruch zur per definitionem gegebenen Offenheit (d. h. mengentheoretischen Unendlichkeit) des Systems, etwa im Bereich der Lexik. Zweitens hat die Verankerung des strukturalistischen Systemkonzepts innerhalb der Sozio- bzw. Variationslinguistik (inklusive der traditionellen philologischen Dialektologie) in Form eines Diasystems (als mengentheoretisch-essentialistisch konfiguriertes System von Systemen) ein weiteres, erkenntnistheoretisch schwerwiegendes Dilemma zur Folge, nämlich das Problem eines Kontinuums, das aus diskreten Varietäten besteht. So etwa im Fall der Annahme einer Menge von Teilmengen, die disjunkt sind, aber gleichzeitig ein Shifting ermöglichen müssen. "Wie will man wirklich Sprachebenen, die fließend ineinander übergehen, oder Systeme, die durch ein Regelsystem miteinander verbunden sind, voneinander trennen und entscheiden, wo welches System beginnt und wo welches aufhört?" (Moosmüller 1987: 32). ${ }^{12}$ Auf Basis der somit erfolgten Charakterisierung der strukturalistisch-linguistischen Systemkonzeption als aus konstruktivistischer Perspektive - Produkt des methodologischen Essentialismus wird nun im Anschluss die linguistische Modellierung von Salienz einer entsprechend fokussierten Kritik unterzogen.

\section{Essentialistische und konstruktivistische Aspekte des linguistischen Salienzkonzepts}

$\mathrm{Zu}$ Recht unterstreicht Lenz (2010: 89) die Bedeutung der kontaktlinguistischdialektologischen Forschungen von Viktor Schirmunski für die Entwicklung des linguistischen Konzepts von Salienz. Die Zahl der einschlägigen Arbeiten, die auf eine Auseinandersetzung mit Schirmunskis Dialektmerkmals-Differenzierung primär/sekundär zurückgehen, ist nahezu Legion (cf. ibd.: 91). Genau an dieser Stelle lassen sich aber nun auch Überlegungen im Sinn einer konstruktivistischen Fruchtbarmachung des Salienzkonzepts (mit dem übergeordneten Ziel einer entsprechenden Potenzierung der Soziolinguistik als Ganzes) entwickeln. Die im Anschluss an Schirmunski beobachtbare Salienz-Diskussion (cf. ibd., insbesondere: 89-95) bewegt(e) sich - was freilich im Licht des vorliegend bislang Ausgeführten wenig verwunderlich ist - über weite Strecken auf einem durch den strukturalistischen Systembegriff

\footnotetext{
${ }^{10}$ Diese klassisch holistische Vorstellung (cf. Popper 1987: 61-66) führt unweigerlich zum Essentialismus: "Man kann den Holismus daher als essentialistischen oder internen Relationalismus bezeichnen" (Bertram/Liptow 2002: 200).

11 "Die Zahl der abstrakt möglichen Relationen zwischen den Elementen eines Systems nimmt exponentiell mit der Zunahme der Zahl der Elemente zu [...]. Wenn in einem System die Zahl der Elemente sehr groß wird, erreicht deshalb die Zahl der Relationen Größenordnungen, die [...] nicht unmittelbar kontrolliert werden können. Das impliziert, daß im System nicht alles [...] zugleich mit allem anderen verbunden werden kann" (Baraldi/Corsi/Esposito 1997: 94).

12 Zum Versuch, unter anderem auch die oben - stark komprimiert - umrissenen Probleme einer Lösung zuzuführen mittels einer dynamischen (panchronischen) Variante des strukturalistischen Systemkonzepts (und somit innerhalb eines noch nicht konsequent, aber bereits in hohem Maß implizit konstruktivistischen Rahmens) cf. Schmidt/Herrgen (2011) und s. Anmerkung 13.
} 
determinierten essentialistischen Terrain, wobei jedoch graduelle (wissenschaftsevolutionär bedingte) Unterschiede auszumachen sind. Die ältere Forschung bzw. die traditionelle philologische Dialektologie repräsentiert eine naturalistische Realismus-Position. Dabei wird vorzugsweise die Objektivität salienter Merkmale diskutiert (und vermeintliche Subjektivität als Defizit klassifiziert, zumindest aber kritisiert (cf. ibd.: 91)). Diese Haltung hat allerdings seit geraumer Zeit eine Ablösung erfahren durch jüngere, kognitivistische/mentalistische Realismus-Strömungen. ${ }^{13}$ Dabei entfallen Werturteile zugunsten so genannter objektiver Merkmale, mehr noch: Plädiert wird beispielweise "für einen Salienzbegriff, der Salienz eindeutig als subjektiv-attitudinale Größe fasst" (ibd.: 89). Daraus folgt allerdings noch keineswegs der entscheidende konstruktivistische Schritt, denn an der Vorstellung der erkenntnistheoretisch relevanten und potenziell empirisch evidenten Korrespondenz dieser Subjektivität mit einer vermeintlichen Objektivität (in der Welt, Wirklichkeit etc.) wird festgehalten (s. oben, Abschnitt 1). Salienz ist zwar ein subjektiv-attitudinales Datum, korrespondiert aber mit "objektiven" Entitäten/Größen. Erst die Überwindung dieses Postulats ermöglicht aber den Anschluss an dezidiert konstruktivistische Positionen. Solche vertreten "die Annahme, daß Erkenntnis nicht auf einer Korrespondenz mit der externen Wirklichkeit beruht, sondern immer nur auf »Konstruktionen« eines Beobachters" (Baraldi/Corsi/Esposito 1997: 100). Objekte (der Wirklichkeit) werden nicht im Sinn einer progressiven Enthüllung exploriert, sondern im Zuge der Erfindung externer Daten (cf. ibd., s. auch oben, Abschnitt 1). Dies führt jedoch keineswegs zu einem negativ zu bewertenden "Verlust" an Realität, denn "die Operationen, welche die Objekte konstruieren, sind genauso real wie die Objekte selbst" (ibd.: 103-104). Damit aber ist der Weg frei für Versuche, das linguistische Salienzkonzept konsequent konstruktivistisch-funktional zu gestalten. ${ }^{14}$

\section{Zur Konstruktion und Zeichenqualität von Salienz}

Innerhalb der Soziolinguistik im weitesten Sinn erfolgte bislang keine nachhaltige Weiterentwicklung des - gemäß Cours-Tradierung - im Saussure'schen Theorierahmen eine elementare Rolle einnehmenden differenzialistischen/differenztheoretischen Ansatzes (s. Anmerkung 7), was eine Anschlussfähigkeit gegenüber modernen systemtheoretischen Paradigmen anbelangt. ${ }^{15}$ Gerade der Salienzbegriff könnte hier als Ausgangspunkt bei der Umsetzung durchaus vielversprechender Intentionen fungieren. Zur Veranschaulichung seien hier folgende Fäden zusammengeführt: a) In den oben mehrmals erwähnten Arbeiten von Schirmunski (cf. 1928/1929: 166) gelten, in typisch strukturalistisch-differenzialistischer Manier, Merkmale "nicht aus sich heraus auffällig" (Lenz 2010: 90), sondern ihre Auffälligkeit ${ }^{16}$ beruht auf einer relationsdeterminierten Kontrastwirkung. - b) Eine differenzialistische Konzeption zieht sich bekanntermaßen auch, z. T. unter expliziter Bezugnahme auf Saussure, als eine Art roter Faden durch das weit verzweigte Universum der Luhmann'schen Systemtheorie (cf. Baecker 2009: 66-91). Der Prozess der Zeichenkonstituierung (als Konstruktion) lässt sich als Unterscheidung (Differenzierung) verstehen, dessen Produkt, das Zeichen (als Konstrukt), wird definiert als die "Einheit der

13 Diese semikonstruktivistische Evolutionsstufe verkörpert anschaulich die sprachdynamisch fundierte Regionalsprachenforschung (cf. Schmidt/Herrgen 2011), und dies bezeichnenderweise auch im Hinblick auf systemtheoretische Implikationen, s. auch Anmerkung 12.

${ }^{14}$ Man beachte, dass es hier nicht darum geht, herauszufinden, was Salienz ("wirklich") ist - dabei würde es sich ja um ein essentialistisches Vorhaben par excellence handeln. Demgegenüber soll konstruktivistisch (und das heißt auch: nominalistisch) vorgegangen werden und der Salienzbegriff ausschließlich über seine Funktionalität definiert und verstanden werden (cf. Popper 1987: 23).

15 Demgegenüber hat sich in der Literaturwissenschaft eine (an die Luhmann'sche Theorie unmittelbar anlehnende) systemtheoretische Denkströmung längst etabliert und voll entfaltet (cf. etwa Werber 2011). Auch generativ-kybernetisch sowie text- bzw. korpuslinguistisch ausgerichtete Positionen innerhalb der Sprachwissenschaft befassen sich mit der Applikation moderner systemtheoretischer Ansätze.

16 Der Terminus Salienz findet in den Arbeiten Schirmunskis noch keine Verwendung. 
Unterscheidung [Differenz, M. M. G.] von Bezeichnendem und Bezeichnetem" (ibd.: 284) .c) Die moderne (semi-)konstruktivistische (cf. Auer 2007: 16) interaktionale Soziolinguistik analysiert soziale Bedeutung als Konstrukt interaktionaler sprachbasierter Operationen, was insbesondere den gesamten attitudinalen, kontextualisierungsrelevanten sowie Stil- und Identitäts-bezogenen Bereich des Gebrauchs natürlicher Sprachen (auf Basis der Funktionalisierung ihrer Heterogenität, d. h. der Funktionalisierung von Differenzierung und Differenz(iertheit)) umfasst. Auf die auch in diesem Zusammenhang entscheidende Bedeutung kontrastbasierter Salienz wurde bereits oben einleitend (Abschnitt 1) hingewiesen. Die systemtheoretische Basis dieser explizit (semi-)konstruktivistischen soziolinguistischen Strömung bleibt allerdings im Wesentlichen noch strukturalistisch bzw. hybrid (s. zum Phänomen der Hybridität von Systemkonzeptionen auch Anmerkung 8) und somit (teils unreflektiert) essentialistisch. - d) Eine herausragende Bedeutung im Zusammenhang mit den hier in einer Zusammenschau einander angenäherten Paradigmen, Strömungen und Positionen nimmt die Weiterentwicklung des linguistischen Salienzkonzepts durch die Forschungen von Christoph Purschke (2011 u. i. Dr.) ein. Darin wird - soweit ich es sehe: erstmals, zumindest innerhalb eines fundierten, stringent argumentierten method(olog)ischen Zusammenhangs der Salienz, in Komplementarität mit dem Konzept Pertinenz, eine semiotisch fundierte teleologische Funktionalität zugewiesen. Purschke argumentiert durchgehend konstruktivistisch, verlässt aber nicht die letztlich strukturalistisch-essentialistische Systembasis des sprachdynamisch fundierten Modells der Regionalsprachenforschung (s. oben). Insbesondere setzt er nicht den naheliegenden letzten Schritt, nämlich Salienz explizit als indexikalisches Zeichen zu behandeln, das die "sozial situative Bedeutung sprachlicher Variationsphänomene" (Purschke i. Dr.) über die Entfaltung von Kontextualisierungsprozessen beeinflusst bzw. (mit-)steuert, und zwar "mit lebensweltlichen Konsequenzen" (ibd.). - e) Diese Option bietet sich im Rahmen des Metasoziosemiose-Konzepts, das eine wesentliche Komponente einer originären - dezidiert und explizit konstruktivistischen - "funktional dimensionierten" soziolinguistischen Theorienbildung auf prozessual-selbstreferenzieller systemtheoretischer Basis darstellt (cf. Glauninger 2012). Unter dem Begriff Metasoziosemiose werden dabei all jene semiotischen Prozesse subsumiert, in deren Verlauf Signata - nämlich (stereotypische) Repräsentationen sprachlicher (Variations-)Phänomene jedweder Art (in Form von / in Verbindung mit sozialen Entitäten bzw. Akkumulationen) - mit sprachbasierten Signantia verknüpft werden. Diese interaktionalen Konstruktionsverfahren produzieren Zeichen vorwiegend indexikalisch kontextualisierender, aber auch - etwa bei hinreichender Konventionalisierung der involvierten Prozesse - symbolischer Art. Das Postulat der Metasoziosemiose schließt ein Festhalten an der essentialistischen Systembasis des Strukturalismus aus schwerwiegenden Gründen aus und ist deshalb eingebettet in den in statu nascendi (bzw. von seinem Selbstverständnis her kontinuierlich in evolutionärer Bewegung) befindlichen Rahmen einer allgemeinen funktional dimensionierten Sprachvariationstheorie. Diese kann hier nur im Hinblick auf den vorliegend behandelten Gegenstand bzw. die im Zusammenhang damit definierte Problemstellung anhand ausgewählter Aspekte umrissen werden: $\alpha$ ) natürlichsprachliche Heterogenität wird nicht durch platoni(sti)sche (wie im Strukturalismus oder Generativismus, s. weiter oben) oder sonstige Homogenisierungsstrategien (cf. Schmidt/Herrgen 2011: 19) method(olog)isch transformiert, sondern als solche dezidiert zum Untersuchungsgegenstand; $-\beta$ ) diese Heterogenität ist des Weiteren wechselseitig Produkt und Medium (im Luhmann'schen Verständnis, cf. Baraldi/Corsi/Esposito (1997: 58-60)) sozialer (Inter-)Aktivität (im weitesten Sinn). Ihre Realität - und selbstredend ganz allgemein die Realität von Sprache(n), Varietäten etc. sowie von sämtlichen damit zusammenhängenden Merkmalen, Kontrasten usw. - ergibt sich ausschließlich aus der Realität ihrer Konstruktion (s. oben). Dies gilt in genau derselben Weise für Salienz; $-\gamma$ ) natürliche Sprache (bzw. ihre Heterogenität) wird - mangels Anwendbarkeit notwendiger Systematizitätskriterien (im modernen systemtheoretischen Sinn) - nicht als 
System behandelt. Ihre systembezogene Analyse erfolgt daher mittels Applikation der soziologischen Systemtheorie als Supertheorie. Sprache bildet in dieser Sichtweise eine Art Mechanismus (Medium, s. oben), der Kommunikationsprozesse in höchstem Maß potenziert, indem er deren kontinuierliche, autopoietisch generierte Sukzession stimuliert und auf diese Weise letztlich sicherstellt. Dies wird insbesondere durch eine spezifisch ausgeprägte Form von Selbstreferenzialität ermöglicht: Erstens weist Sprachliches als solches innersprachlich Zeichenqualität auf; und zweitens kann Sprachliches ausschließlich sprachlich beobachtet (und kommuniziert) werden, und zwar in sämtlichen sozialen Kontexten - insbesondere auch (sprach-)wissenschaftlich. Spätestens im Licht dieses Faktums, dem die moderne Systemtheorie mit der Verortung des Beobachters innerhalb des Systems Rechnung trägt, erweisen sich sämtliche Postulate einer "objektiven" wissenschaftlichen Beobachtung (und Beschreibung) von Sprache durch ein epistemisches Subjekt von "außen" - oder von einem archimedischen Punkt aus -, etwa in Form einer cartesianischen Linguistik, aber auch mithilfe einer vermeintlich nicht natürlichsprachlich determinierten Metasprache (z. B. auf Basis eines formalen Kalküls), ebenso als Illusion wie jedwede andere Annahme "objektiver" wissenschaftlicher Beobachtung und Beschreibung, in welcher Disziplin auch immer. ${ }^{17}$ Der systemtheoretische Beobachter innerhalb des Systems bildet somit gewissermaßen das Pendant soziologischer (und bei Applikation: linguistischer) Methodologie $\mathrm{zu}$ entsprechenden Verfahren innerhalb der Naturwissenschaften (etwa der Behandlung der Unschärferelation gemäß Heisenberg). Eingebettet in einen dergestalt modellierten - hier freilich auf wenige Aspekte komprimierten - theoretischen Zusammenhang lässt sich somit Salienz interpretieren als (indexikalisches) Zeichen, das innerhalb des komplexen Feldes (der Konstruktion) sozialer Bedeutung auf vielfältige Weise Wirksamkeit entfaltet.

\section{$5 \quad$ Resümee}

Der innerhalb innovativer (post-)moderner (etwa interaktionaler oder sprachdynamisch fundierter) soziolinguistischer ${ }^{18}$ Paradigmen gebräuchliche, überwiegend essentialistische Salienzbegriff indiziert deren nicht weniger essentialistische (weil nach wie vor strukturalistische) Systemkonzeption. Diese Diagnose des epistemologischen Status der gegenwärtigen Soziolinguistik, resultierend aus einer im vorliegenden Beitrag unternommenen, dezidiert und explizit konstruktivistisch positionierten Analyse, soll zu einer breiteren Diskussion anregen. Eine konstruktivistische Transformation des linguistischen Salienzkonzepts könnte die Anschlussfähigkeit der gegenwärtigen (Sozio-)Linguistik gegenüber zeitgemäßen, prozessual-selbstreferenziell modellierten systemtheoretischen Paradigmen (zumindest punktuell) erhöhen. Für eine entsprechend konfigurierte Soziolinguistik ist Salienz ein Konstrukt der Kommunikation/Interaktion, ein Zeichen, das wie andere Zeichen auch - und zusammen mit diesen - soziale Bedeutung konstituiert bzw. gleichzeitig von dieser determiniert wird. Die Realität von Salienz (und von entsprechenden sprachlichen Merkmalen) entspricht der Realität der interaktionalen Konstruktionsprozesse, deren Produkte sie sind. Prozessual-selbstreferenziell modellierte Systemkonzepte sind dazu geeignet, Problemerfahrungen, die mit Phänomenen dieser Art - d. h. letztlich mit der Heterogenität natürlicher Sprache(n) - verbunden sind, hinreichend konsistent zu organisieren bzw. zu strukturieren und dadurch für unterschiedliche wissenschaftliche Diskurse fruchtbar zu machen.

\footnotetext{
17 Diese Haltung ist in den modernen Naturwissenschaften spätestens seit der Quantentheorie als Standard etabliert, s. weiter oben, Anmerkung 6.

${ }^{18}$ Darunter fallen natürlich auch die Variations- und Perzeptionslinguistik.
} 


\section{Literatur}

Auer, Peter (ed.) (2007): Style and Social Identities. Alternative Approaches to Linguistic Heterogeneity. Berlin/New York: de Gruyter. (=Language, Power and Social Process 18).

Baecker, Dirk (ed.) (2009): Niklas Luhmann. Einführung in die Systemtheorie. 5. Auflage. Heidelberg: Auer.

Bally, Charles/Sechehaye, Albert (eds.) (2001): Ferdinand de Saussure. Grundfragen der allgemeinen Sprachwissenschaft. 3. Auflage. Berlin/New York: de Gruyter.

Baraldi, Claudio/Corsi, Giancarlo/Esposito, Elena (1997): GLU. Glossar zu Niklas Luhmanns Theorie sozialer Systeme. Frankfurt a. M.: Suhrkamp. (= Suhrkamp Taschenbuch Wissenschaft 1226).

Berg, Thomas (1998): Linguistic Structure and Change. An Explanation from Language Processing. Oxford etc.: University Press.

Bertram, Georg W./Liptow, Jasper (eds.) (2002): Holismus in der Philosophie. Ein zentrales Motiv der Gegenwartsphilosophie. Weilerswist: Velbrück.

Besch, Werner et al. (eds.) (1998): Sprachgeschichte. Ein Handbuch zur Geschichte der deutschen Sprache und ihrer Erforschung. 2., vollständig neu bearbeitete und erweiterte Auflage. Berlin/New York: de Gruyter. (= Handbücher zur Sprach- und Kommunikationswissenschaft 2.1).

Chomsky, Noam (2002): On Nature and Language. With an essay on "The secular priesthood and the perils of democracy". Cambridge etc.: University Press.

Deppermann, Arnulf (2007): Grammatik und Semantik aus gesprächsanalytischer Sicht. Berlin/New York: de Gruyter. (= Linguistik-Impulse \& Tendenzen 14).

Glauninger, Manfred M. (2012): "Zur Metasoziosemiose des 'Wienerischen'. Aspekte einer funktionalen Sprachvariationstheorie". Zeitschrift für Literaturwissenschaft und Linguistik 166: 110-118.

Heisenberg, Werner (1990): Physik und Philosophie. Frankfurt a. M./Berlin: Ullstein. (= Neuauflage des Ullstein-Materialien-Bandes 35132, = Ullstein Sachbuch. Ullstein Buch 34624).

Lenz, Alexandra Nicole (2010): "Zum Salienzbegriff und zum Nachweis salienter Merkmale". In: Anders, Christina A./Hundt, Markus/Lasch, Alexander (eds.): Perceptual Dialectology. Neue Wege der Dialektologie. Berlin/New York, de Gruyter: 89-110. (= Linguistik Impulse \& Tendenzen 38).

Luhmann, Niklas (1987): Soziale Systeme. Grundriß einer allgemeinen Theorie. Frankfurt a. M.: Suhrkamp. (= Suhrkamp Taschenbuch Wissenschaft 666).

McMahon, April M. S. (1999): Understanding Language Change. Cambridge etc.: University Press.

Moosmüller, Silvia (1987): Soziophonologische Variation im gegenwärtigen Wiener Deutsch. Eine empirische Untersuchung. Stuttgart: Steiner. (= ZDL-Beihefte 56).

Payne, Michael/Barbera, Jessica Rae (eds.) (2010): A Dictionary of Cultural and Critical Theory. 2. Auflage. Malden/Oxford: Wiley-Blackwell.

Popper, Karl Raimund (1987): Das Elend des Historizismus. 6., durchgesehene Auflage. Tübingen: Mohr. (= Die Einheit der Gesellschaftswissenschaften. Studien in den Grenzbereichen der Wirtschafts- und Sozialwissenschaften 3).

Purschke, Christoph (2011): Regionalsprache und Hörerurteil. Grundzüge einer perzeptiven Regionalsprachenforschung. Stuttgart: Steiner. (= Zeitschrift für Dialektologie und Linguistik Beihefte 149).

Purschke, Christoph (i. Dr.): Wort und Totschlag. Zur sozio-symbolischen Bedeutung sprachlicher Divergenz.

Raimy, Eric/Cairns, Charles E. (eds.) (2009): Contemporary Views on Architecture and Representations in Phonology. Cambridge, MA: MIT Press. 
Schirmunski, Viktor M. (1928/1929): "Die schwäbischen Mundarten in Transkaukasien und Südukraine". Teuthonista 5: 38-60, 157-171.

Schmaus, Warren (1994): Durkheim's Philosophy of Science and the Sociology of Knowledge. Creating an Intellectual Niche. London/Chicago: University of Chicago Press.

Schmidt, Jürgen Erich/Herrgen, Joachim (2011): Sprachdynamik. Eine Einführung in die moderne Regionalsprachenforschung. Berlin: Schmidt. (= Grundlagen der Germanistik 49).

Werber, Niels (ed.) (2011): Systemtheoretische Literaturwissenschaft. Begriffe - Methoden Anwendungen. Berlin/New York: de Gruyter. (= De Gruyter Lexikon). 